\title{
Multiresolution Layered Manufacturing
}

First author address:

Department of Computer Sciences and Languages, University of Málaga.

Bulevar Louis Pasteur, 35, 29071 Málaga, Spain.

Email: josedavid@uma.es

Second author address:

Laboratory of Physics of Surfaces and Interfaces, University of Mons.

Avenue Victor Maistriau, 19, 7000 Mons, Belgium.

Email: joel.deconinck@umons.ac.be

Corresponding author: first author.

\section{Abstract}

Purpose

Two-photon polymerization (TPP) has become one of the most popular techniques for stereolithography at very high resolutions. When printing relatively large structures at high resolutions, one of the main limiting factors is the printing time. The goal of this work is to present a new slicing algorithm to minimize printing times.

Design/methodology/approach

Typically, slicing algorithms used for TPP do not take into account the fact that TPP can print at a range of resolutions (i.e. with different heights and diameters) by varying parameters such as exposure time, laser power, photoresist properties, and optical arrangements. This work presents Multiresolution Layered Manufacturing (MLM), a novel slicing algorithm that processes 3D structures to separate parts manufacturable at low resolution from those that require a higher resolution.

\section{Findings}

MLM can significantly reduce the printing time of 3D structures at high resolutions. The maximum theoretical speed-up depends on the range of printing resolutions, but the effective speed-up also depends on the geometry of each 3D structure.

\section{Research limitations/implications}

MLM opens the possibility to significantly decrease printing times, potentially opening the use of TPP to new applications in many disciplines such as microfluidics, metamaterial research or wettability.

\section{Originality/value}

There are many instances of previous research on printing at several resolutions. However, in most cases, the toolpaths have to be manually arranged. In some cases, previous research also automates the generation of toolpaths, but they are limited in various ways. MLM is the first algorithm to comprehensively solve this problem for a wide range of true 3D structures.

Keywords

two-photon polymerization, additive manufacturing, 3D printing, slicing, multiresolution 


\section{Introduction}

Technology and science improve together, as new science enables new technologies, and vice versa. As part of this virtuous cycle, researchers have developed technologies that enable the manufacturing of objects and surfaces with very small features in the micron and sub-micron precision range, such as patterning processes (for example, photolithography), additive manufacturing and self-assembly processes. From these technologies, additive manufacturing stands out as giving very precise control over the shape of the object or surface to manufacture, but has a critical problem: it is very slow when the object has a big volume relative to the printing resolution.

These technologies have the potential to enable new research in multiple disciplines. Just to quote a few, let us point out wettability and more particularly the lotus effect where a double scale roughness surface is preferable (De Coninck et al., 2015). On top of such surfaces, it is expected that the drop can be in at least two different states: in contact everywhere with the solid surface, i.e. the so-called wet or Wenzel state, or in contact with only the top elements of the surface, the so called dry or CassieBaxter state. Superhydrophobicity or lotus effect refers naturally to this Cassie-Baxter state and may well lead to myriads of technical applications.

Another example is given by bone reconstruction where porous biomaterials are designed to assist or replace organ functions and improve quality of life. It is a real challenge and corresponds to a societal need. Indeed, the first generation of biomaterials was developed from materials designed originally for engineering applications. Although these biomaterials provide an effective immediate solution for many patients, the outcome is often time-limited. As a consequence, considerable research interest has focused on investigating mechanisms that contribute to implant-prosthesis failure and on developing new biomaterials with an extended lifetime and extended biocompatibility properties where multiscale surfaces play a key role (Delattre et al., 2014).

\subsection{Additive manufacturing}

There has been considerable interest in developing and refining additive manufacturing (AM) technologies over the last few decades (Huang et al., 2013). Typically, AM works by taking a computer 3D model, slicing it along its $\mathrm{Z}$ axis, and configuring a sequence of machine movements that effectively print the slices one after another. These tasks are performed by slicing software. Without slicing software, machine operators have to manually configure the toolpaths, which can become quickly unwieldy for even relatively simple geometries. Therefore, for many use cases, slicing software is almost a prerequisite for $\mathrm{AM}$.

Currently, for most off-the-shelf software, slicing algorithms are implemented assuming that printing parameters such as the resolution are constant for each printing job. Usually, there is a trade-off between speed and quality of printing, with printing resolution being one of the most outstanding parameters that influence this trade-off. This work introduces a new slicing algorithm that is able to keep high resolution features with faster printing times, for systems able to do AM at several resolutions. This may be achieved by using several different technologies (hybrid manufacturing, Chu et al. 2014) or a technology able to print at several resolutions, such as two-photon polymerization, the latter being the one used in the experimental setup described in Section 2.3. 


\subsection{Two-photon polymerization}

Two-photon polymerization (TPP) is a technology for 3D microfabrication in which a photoresist (a mix of a photosensitizer and a polymerizable resin in liquid or gel state) is irradiated with a femtosecond-pulsed infrared laser beam which is highly focused by a microscope objective (Sun and Kawata, 2004; Spangenberg et al., 2013). The photosensitizer and the laser are engineered such that the photosensitizer must simultaneously absorb two photons from the laser beam in order to become active. Because of this, a very large influx of photons is necessary to start the polymerization reaction, thus the laser beam polymerizes the resin only in a small region around the focal point, smaller than the wavelength of the laser.

Typically, the photoresist is put over a flat substrate, which is mounted in very high precision stages in the $\mathrm{X}, \mathrm{Y}$ and $\mathrm{Z}$ axes (width, depth and height, resp.) in order to move the focal point relative to the substrate. In this way, lines can be polymerized over the substrate. After all lines are written, the leftover photoresist is washed away in a development process. In some machines, very fast movements over a small area on the XY plane can be achieved using galvanometer-driven scanning mirrors (galvos) to deflect the laser beam. Galvos can be used to print several orders of magnitude faster than using stages, but with less precision, over narrower ranges, and only for constant-height lines.

The region polymerized around the focal point is the voxel. In TPP, the voxel is a prolate spheroid whose main axis coincides with the optical axis of the objective. Its shape and size depends on laser intensity, exposure time, the optical properties of the objective (Sun et al., 2003), and the chemical, optical and physical properties of the photoresist. Thus, modifying these, it is possible to change the size of the voxel, and consequently the printing resolution. Large vertical size ranges have been reported, from $0.6 \mu \mathrm{m}$ to $15 \mu \mathrm{m}$ (Lim et al., 2006). However, no slicing software package readily available can take advantage of this characteristic. Currently, users must define manually the toolpaths if they want to print at several resolutions.

\subsection{Related work}

TPP is very slow when it is used to manufacture relatively large objects at high resolution. This problem has been addressed in multiple ways, such as adapting manufacturing equipment and methods (hardware): using digital micro-mirror devices to cure whole resin layers at once ( $\mathrm{Li}$ et al., 2012), or using arrays of micro-lenses (Matsuo et al., 2005) or spatial light modulation techniques (Gittard et al., 2011) to cast multiple replicas of a single pattern. The problem is more about the size of the voxel than the specific characteristics of TPP, so it affects other manufacturing technologies at very high resolution, such as laser micromachining.

Another way to increase printing speed is to modify the way toolpaths are generated or arranged. For example, Lim et al. (2008) shortened printing times by organizing the toolpaths to achieve higher average speeds. In some cases, some slicing methods were developed earlier for TPP than for other technologies, such as non-horizontal slicing (Liao et al., 2007; Hildebrand et al., 2013), because TPP is not constrained to work layer by layer as other methods (truly 3D lines can be written with TPP, but previously written parts can distort the laser beam, reducing manufacturing quality if the ordering of the toolpaths is not carefully planned). In other cases, researchers borrow or adapt relatively old slicing techniques developed for other technologies, as Park et al. (2005), who effectively applied variable-width (adaptive) slicing (Pandey et al., 2003) to TPP. 
The present work is in a similar position, since Ma and He (1999) already proposed a method for adaptively slicing B-spline surfaces, which is effectively the same as using several different resolutions. The scope of this work is different, though: it is more centered on the issue of multiresolution slicing as opposed to specifically slicing Bspline surfaces, and the algorithm presented here is more generic.

The idea of using several different manufacturing technologies to shorten fabrication times is also hardly new; there is a large body of previous work on hybrid manufacturing technologies (Chu et al., 2014; Zhu et al., 2013). Focusing on the combination of different technologies for high-resolution layered manufacturing, there have been efforts to do additive/additive combinations, such as UV stereolithography and TPP (Eschenbaum et al., 2013), additive and subtractive TPP (Lim et al., 2011; Xiong et al., 2012), FFF (fused filament fabrication) and subtractive direct laser writing (Malinauskas et al., 2014), FFF and TPP (Balčiūnas et al., 2014), and TPP at several different resolutions (Lim et al., 2006; Jonušauskas and Malinauskas, 2014).

However, most of this research has focused on devising procedures and protocols to overcome the barriers to combine different manufacturing technologies. In almost all the previously mentioned works, the user still has to manually plan how to arrange the toolpaths at different resolutions. While this is feasible for simple and small objects or patterns, it quickly becomes impractical when it comes to bigger and more complex or non-regular ones. This is the purpose of this work: given a sequence of manufacturing technologies, the algorithm is designed to automatically detect the parts of the model that can be manufactured at each resolution. As printing at low resolution is usually much faster than printing at high resolution, the goal is to significantly shorten the printing time with respect to printing everything at high resolution.

There have been some works similar in scope to this one, such as the Multi-Voxel Matrix method of Lim et al. (2006), able to do this automatic separation of an object into several parts by resolution, but it is adequate only for thin objects with no overhangs, while the algorithm presented here has no such limitations.

\section{Methods}

Slicing software is usually organized as a pipeline with the following stages:

1. STL file loading (3D structure as a triangle mesh)

2. Analysis and repairing of the triangle mesh

3. Slicing

4. Toolpath generation

5. Motion planning

6. G-code generation

Steps 1 to 3 were reused from a popular open-source project, Slic3r (Ranellucci, 2017). This work is focused in the third step (slicing proper). In it, the $3 \mathrm{D}$ structure is intersected with horizontal planes, to get a sequence of slices that approximate the 3D structure. The contribution of this work is an algorithm to process and modify these slices in step 3. Steps 4 to 6 are also important, but they are not the subject of this study, so basic but reasonably efficient implementations were written to complement the slicing algorithm. 


\subsection{Multiresolution Layered Manufacturing}

The simplest slicing method is fixed slicing, where the slicing planes are defined at constant intervals. The alternative to this is adaptive slicing (Sabourin et al., 1996; Ma and He, 1999; Park et al., 2005), which can be used if the underlying manufacturing technology is compatible with overlapping toolpaths. This is the case of TPP: already solidified volumes can be re-exposed to the laser beam. With adaptive slicing, resolution in the vertical axis can be lower than the thickness of the slices, although with an important limitation: no overhanging feature can be thinner than the slice thickness (these are 2.5D features, since they can be expressed as a height function over a $2 \mathrm{D}$ domain. Also, to apply adaptive slicing in an effective way, the steepness profile of the $3 \mathrm{D}$ structure must be computed across the vertical axis, so that the spacing of the slices is inversely correlated with the steepness of the shape.

Usually, both fixed slicing and adaptive slicing assume a constant voxel size and shape. In contrast, this work describes Multiresolution Layered Manufacturing (MLM), an algorithm to define slices from a 3D structure using two or more voxel types, each one with a different size and shape. For simplicity and economy of presentation, MLM is presented as a fixed slicing strategy (i.e. each voxel type defines a set of constantspaced slices), but it may also be implemented as an adaptive slicing strategy.

The basic idea behind MLM is to work iteratively from lower to higher resolutions, repeatedly carving out of the 3D structure the parts that can be printed at each resolution. To apply MLM, $n$ voxel types $(n \geq 2)$ are defined, characterized by their monotonically decreasing heights $\triangle z_{1} \leqslant \ldots \leqslant \triangle z_{n}$ and maximal horizontal diameters $\triangle x_{1} \leqslant \ldots \leqslant \triangle x_{n}$ (i.e. ordered from lowest to highest resolution). For TPP, all voxels are prolate spheroids.

To slice a 3D structure, a sequence of input slices $s_{i 0}, s_{i 1}, s_{i 2}, \ldots$ is defined for each voxel type $i$. Each $s_{i j}$ is a set of horizontal contours, the result of intersecting the 3D structure with a horizontal plane at height $z_{i j}$. If the 3D structure extends in the vertical axis from $z_{A}$ to $z_{B}$, each $z_{i j}$ is defined as:

$$
z_{i j}=z_{A}+\triangle z_{i}(j+0.5), 0 \leq j \leq\left\lfloor\frac{z_{B}-z_{A}-\triangle z_{i}}{\triangle z_{i}}\right\rfloor
$$

For each input slice $s_{i j}$, two associated sets of higher and lower resolution neighbors $H_{i j}^{s}$ and $L_{i j}^{s}$ are defined, as the respective sets of slices of higher/lower resolution that overlap with $s_{i j}$ in $\mathrm{Z}$ :

$$
\begin{aligned}
H_{i j}^{s} & =\left\{s_{k l}: i<k,\left|z_{i j}-z_{k l}\right|<\left|\left(\triangle z_{i}+\triangle z_{k}\right) 0.5\right|\right\} \\
L_{i j}^{s} & =\left\{s_{k l}: i>k,\left|z_{i j}-z_{k l}\right|<\left|\left(\triangle z_{i}+\triangle z_{k}\right) 0.5\right|\right\}
\end{aligned}
$$

Note that any of these sets $H_{i j}^{s}$ or $L_{i j}^{s}$ can be empty. The slices are processed using set operations (union, intersection, and difference between two slices) and morphological operations (dilation and erosion). The notation $a \oplus b(r)$ is used for the dilation of the slice $a$ with a circle of radius $r$, and $a \ominus b(r)$ for the equivalent erosion.

MLM processes each input slice $s_{i j}$ into the definitive slice $d_{i j}$ with the following specification:

- If $s_{i j}$ were printed as is (with voxels of type $i$ ), some parts of it may protrude from the intended shape (see red dashed shape in Figure 1, d). To avoid this, all parts of $s_{i j}$ that do not overlap with all members of $H_{i j}^{s}$ must be removed, but $s_{i j}$ and the members of $H_{i j}^{s}$ are not directly comparable, as the extent of the slice $s_{i j}$ is 


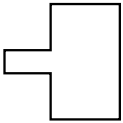

(a)

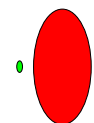

(b)

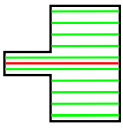

(c)

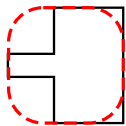

(d)

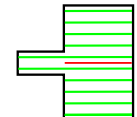

(e)

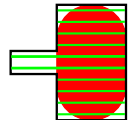

(f)

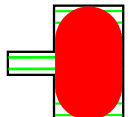

(g)

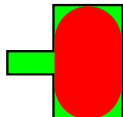

(h)

Fig. 1: Simplified 2D example of the MLM slicing algorithm, see Section 2.1 for details.

not constant across its vertical extent. To make them comparable, $H_{i j}^{o}$ is defined as the set of offset-corrected higher-resolution neighbors, each one dilated by the amount that $s_{i j}$ is shrunk at each height:

$$
H_{i j}^{o}=\left\{o_{k l}=s_{k l} \oplus b(r): s_{k l} \in H_{i j}^{s}, r=x_{i}-p_{i}\left(z_{k l}-z_{i j}\right)\right\}
$$

Where $p_{i}(d)$ is the horizontal extent of the voxel at a vertical distance $d$ from its center.

- On the other hand, high resolution slices should not include parts that have already been filled at lower resolutions (see Figure 1, f). To avoid this, all parts of $s_{i j}$ that overlap with other already printed parts must be removed. These parts to be removed are the set of neighboring lower-resolution definitive slices:

$$
L_{i j}^{d}=\left\{d_{k l}: s_{k l} \in L_{i j}^{s}\right\}
$$

However, $s_{i j}$ is not directly comparable with members of $L_{i j}^{d}$, for the same reasons as in the previous point. To make them comparable, $L_{i j}^{o}$ is defined as the set of offset-corrected lower-resolution definitive slices, each one eroded to match its effective size at the level $z_{i j}$ :

$$
L_{i j}^{o}=\left\{o_{k l}=d_{k l} \ominus b(r): d_{k l} \in L_{i j}^{d}, r=x_{i}-p_{i}\left(z_{i j}-z_{k l}\right)\right\}
$$

- With the previous definitions, each definitive slice $d_{i j}$ is computed operating $s_{i j}$ with the members of $H_{i j}^{o}$ and $L_{i j}^{o}$ (projected onto the plane of $s_{i j}$ ):

$$
d_{i j}=\left(s_{i j} \cap\left(\bigcap_{o_{k l} \in H_{i j}^{o}} o_{k l}\right)\right)-\left(\bigcup_{o_{k l} \in L_{i j}^{o}} o_{k l}\right)
$$

Note that the second part of the equation requires all members of $L_{i j}^{d}$ to be already computed, so the computation order cannot be arbitrary.

With this definition, each definitive slice $d_{i j}$ extends over as much as possible of the original shape, but without overwriting higher resolution overhanging features, and without overwriting what has already been printed at lower resolutions. We can see a graphical example in Figure 1, a-h: a structure (1.a) is to be printed with two voxel types (1.b): high-resolution (small voxel, green) and low-resolution (big voxel, red). In its simplest form, a fixed slicing of the structure is computed for each voxel type (1.c). The only low-resolution slice, if printed as is, would overprint the object (red dashed shape in 1.d). To avoid this, the low-resolution slice is intersected with all highresolution slices near it (1.e). The result is that the bulk of the structure is printed faster, 
at low-resolution (1.f). The high-resolution slices overlap the low-resolution volume, though. To correct this, the already printed space for the low-resolution slice is removed from each high-resolution slice (1.g) to restrict them to high-resolution features (1.h).

Many additional tweaks can be applied to the original algorithm, such as:

- Make sure to remove from the definitive slices any part that is hanging free without any support.

- Extend toolpaths for high resolution features slightly into the (low resolution) bulk of the structure, to ensure robust attachment of these features.

- Enable the user to define parts of the 3D structure where high resolution is not required, so they can be skipped from computations and actual printing.

- Implement adaptive slicing for a more effective reproduction of the original shape.

\subsection{Speed-ups}

As MLM prints as much from a 3D structure as it can at low resolution, it minimizes the printing time. The question is to quantify the speed-up. As a first approximation, AM technologies spend time approximately proportional to the total length of the toolpaths, disregarding effects such as the possible need to modulate printing speed depending on toolpath geometry, and the time spent to go from the end of each toolpath to the beginning of the next one in the sequence. In more formal terms, if:

- each voxel type $i$ has an effective printed volume $m_{i}$,

- and can be printed at speed $p_{i}$ (measured in the number of voxel volumes printed per second ${ }^{1}$ ),

- and a 3D structure has a volume $V$,

- and MLM partitions the 3D structure into sub-volumes $v_{1}+\ldots+v_{n}=V$ (each sub-volume corresponding to a voxel type ${ }^{2}$ ),

then, the speed-up with respect to printing the whole 3D structure at high resolution will approximately be:

$$
\text { speed-up }=\left(\frac{V}{m_{n} p_{n}}\right)\left(\sum_{i=1 \ldots n} \frac{v_{i}}{m_{i} p_{i}}\right)^{-1}
$$

In this way, 3D structures with a comparatively small volume of high resolution features will be printed considerably faster than if printed only at the highest resolution. The bigger the difference between the minimal and maximal voxel sizes, the larger the speed-up. If all printing speeds are identical, speed-ups range from 1 (i.e. actually no speed-up) for 3D structures allowing toolpaths only at the highest resolution, to an upper limit of $m_{1} / m_{n}$ for $3 \mathrm{D}$ structures that can be printed entirely at the lowest resolution.

\footnotetext{
${ }^{1}$ This is mostly equivalent to measuring it in printed toolpath length per second, if the cross-sectiona dimensions of most toolpaths are much smaller than their lengths.

2 This is an approximation, since (depending on the configuration) there will be (mostly negligible) gaps and/or overlaps between the printed toolpaths.
} 
This picture can become more complex for some AM technologies, as it is the case of TPP, where it is common to use galvos for XY toolpaths, and a $\mathrm{Z}$ stage (several orders of magnitude slower) to move in the vertical axis. As a result, the fewer nonempty high resolution slices (i.e. the fewer distinct heights at which high resolution features are required), the faster the printing; the actual total length of toolpaths at each resolution having relatively little effect on the actual speed-up. The upper speed-up limit is the same as in the simpler case, though, since it simply represents the limit at which no high resolution toolpaths are required.

\subsection{Experimental setup}

To test MLM, a commercial TPP 3D printer is used: a Photonic Professional GT from Nanoscribe $\mathrm{GmbH}$. As it is usual for commercial providers of cutting-edge AM technologies, the accompanying slicing software cannot be modified or extended, but the machine also accepts sequences of toolpaths in its own scripting language (GWL script). A post-processing stage can be added to the software pipeline so the toolpaths generated by MLM are translated to GWL.

This machine has several modes of operation. In the experimental setup described here, it is used in DiLL configuration (Dip-in Laser Lithography). Typically, when using objectives with high NA (Numerical Aperture), immersion oil is used to achieve optimal focusing through the substrate. However, in DiLL, the objective is in direct contact with a conveniently index-matched photoresist (IP-Dip, developed by the same company), upside-down with respect to typical substrate configurations. To keep the experimental setup simple, only one objective (x63, NA 1.4) is used: the one recommended by Nanoscribe to create the smallest possible voxels in DiLL configuration. Using the galvos, the printing speed is set to $10000 \mu \mathrm{m} / \mathrm{s}$. The maximal effective laser power delivered through the objective in this configuration is $50 \mathrm{~mW}$, but the effective dose can be modulated.

The application of MLM requires to reliably print with at least two different voxel sizes. Testing the experimental setup, the minimal voxel height in this configuration was found to be around $0.6 \mu \mathrm{m}$ (at $9 \mathrm{~mW}$ ) with isolated toolpaths (if toolpaths are close enough, the higher radiation dose increases the effective voxel size). Maximal voxel height was around $2.25 \mu \mathrm{m}$ (at $40 \mathrm{~mW}$ ) with isolated toolpaths (if they are close enough, the higher radiation dose induces localized explosions that severely degrade the printing quality). To achieve better reliability and replicability, the experiments are carried out with a slightly bigger minimal voxel size, around $0.75 \mu \mathrm{m}$ high and $0.5 \mu \mathrm{m}$ wide (at $11 \mathrm{~mW}$ ), as well as a smaller maximal voxel size, around $1.6 \mu \mathrm{m}$ high and $0.7 \mu \mathrm{m}$ wide (at $30 \mathrm{~mW}$ ).

For practical reasons, the layer height of the maximal voxels is set at $1.5 \mu \mathrm{m}$ instead of $1.6 \mu \mathrm{m}$, to make for a more effective bonding between layers and minimize unpolymerized resin between the layers (for the minimal voxels, this is less critical, as there will be significant amounts of unpolymerized resin, anyway). These minimal and maximal voxel sizes will represent two voxel types (in the sense of Section 2.1). While more than two voxel sizes may be used (i.e., add intermediate sizes), it would make the tests more complex for little practical gain, since the maximal voxel is just about $2 \mathrm{X}$ the size of the minimal one.

The upper speed-up limit is $2 \mathrm{X}$ in this configuration. This limit holds even considering the use of galvos for XY movement and a $\mathrm{Z}$ stage for vertical movements (see Section 2.2), because the difference between both voxel shapes is mostly in the vertical 


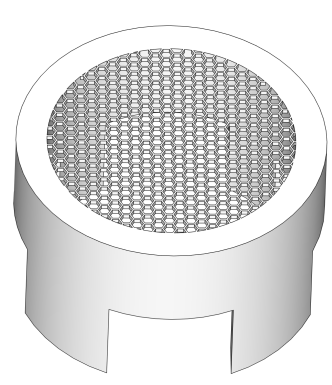

(a.1)

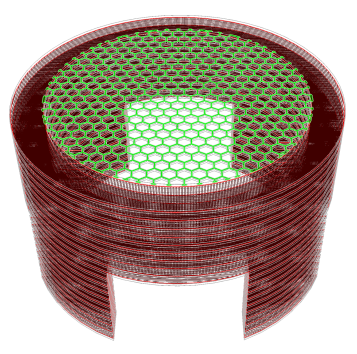

(b.1)

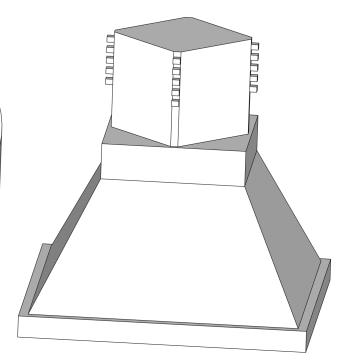

(a.2)

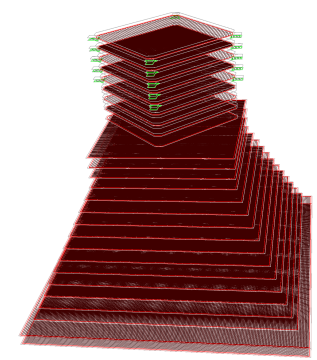

(b.2)

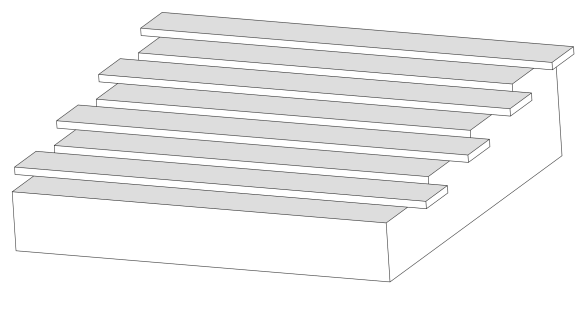

(a.3)

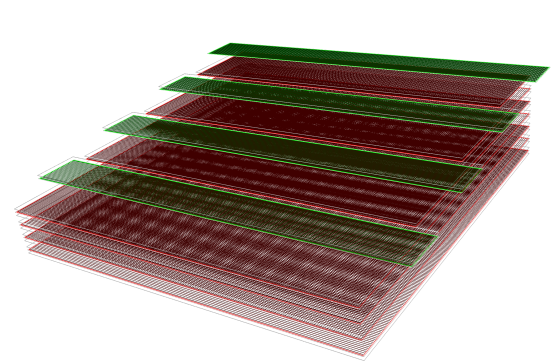

(b.3)

Fig. 2: Three examples of 3D structures; see also Figure 3.

direction, so the difference in size translates into a very similar difference in amount of slices.

\section{Results}

Three 3D structures were selected to test the MLM algorithm:

- A filter-like element (Figure 2, a.1) with an overall diameter of $50 \mu \mathrm{m}$ and hexagonal holes $2 \mu \mathrm{m}$ in diameter.

- A tower with small periodic overhanging features (Figure 2, a.2). These features are $0.75 \mu \mathrm{m}$ high and $1 \mu \mathrm{m}$ deep and wide, while the base is a $40 \mu \mathrm{m}$ square, and the top has a width (from overhang to overhang) of $21 \mu \mathrm{m}$

- A staircase with overhanging steps (Figure 2, a.3). It has a square base of $40 \mu \mathrm{m}$, stairsteps $0.75 \mu \mathrm{m}$ high and $5 \mu \mathrm{m}$ wide, and overhangs $2 \mu \mathrm{m}$ deep.

A software pipeline developed to implement MLM was used to generate two different types of GWL scripts:

- Type A, using both voxel sizes (shown in Figure 2, row b): low-resolution (red toolpaths, voxel around $1.5 \times 0.7 \mu \mathrm{m}$ ) and high-resolution (green toolpaths, voxel around $0.75 \times 0.5 \mu \mathrm{m}$ ). In the case of the toolpaths for the tower (Figure 2, b.2), the software was configured to avoid printing high-resolution toolpaths in the frustum. Examples of these structures are shown in Figure 3, row c.

- Type B, using only small voxels, in order to compare their printing times. These structures are not shown here. 

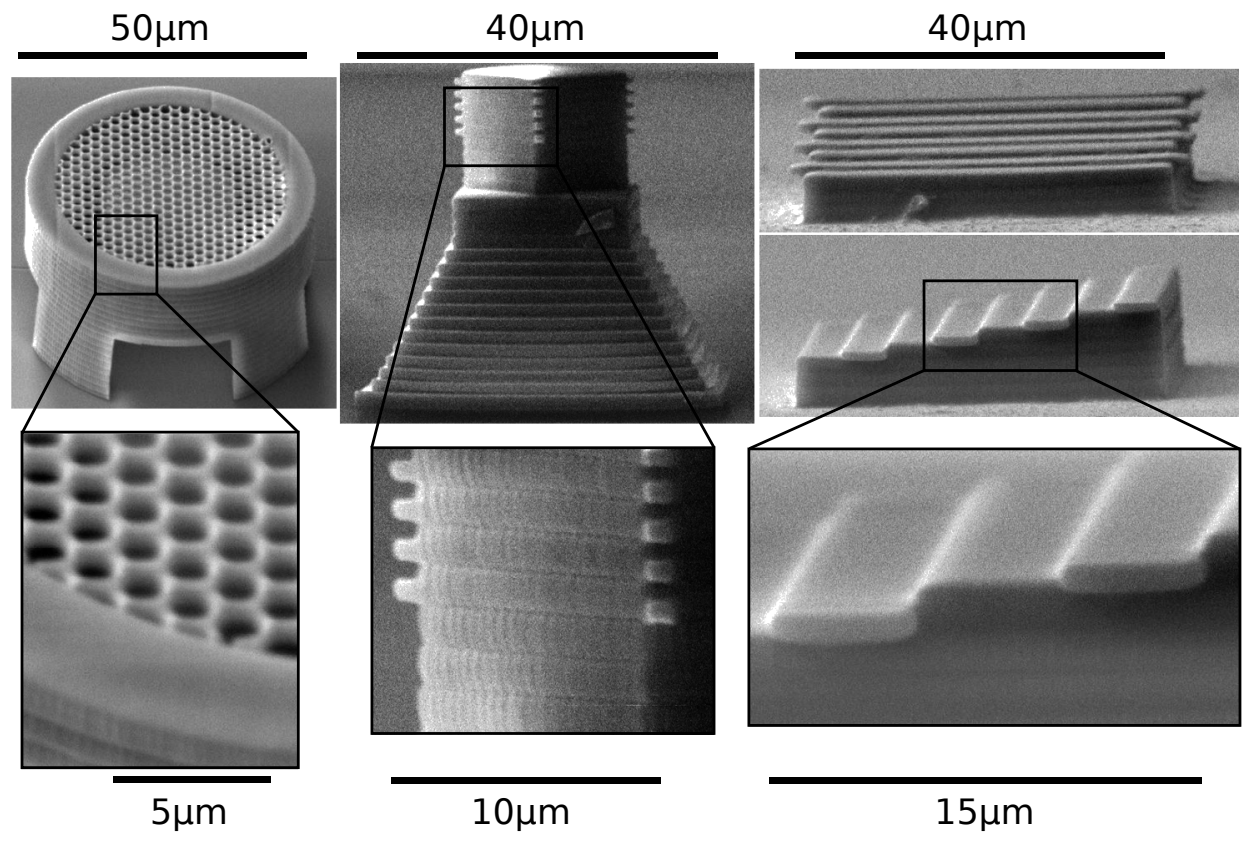

(c.1)

(c.2)

(c.3)
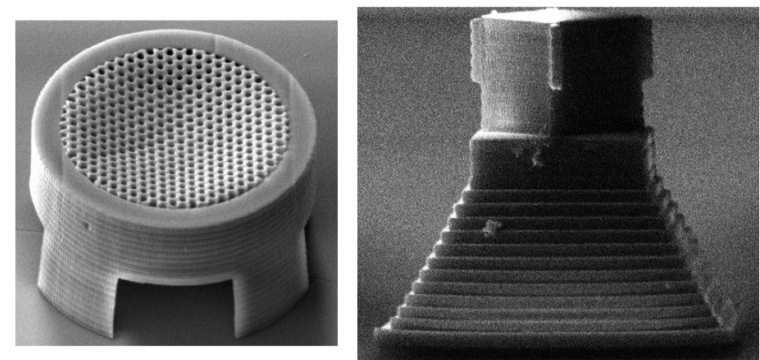

(d.1)

(d.2)

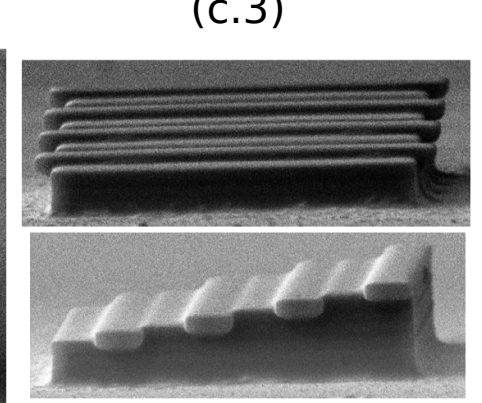

(d.3)

Fig. 3: Continuation of Figure 2. 
Tab. 1: Information about printed structures.

\begin{tabular}{llllllll}
\hline structure & base & overhangs & slices & slices & printing time & printing time & speed-up \\
Figure 2, row a & $\mathrm{Z} \times \mathrm{X}$ & $\mathrm{Z} \times \mathrm{X} \times \mathrm{Y}$ & type A & type B & type A & $\begin{array}{l}\text { type B } \\
\text { B } / \mathrm{A}\end{array}$ \\
\hline a.1: filter & $30 \times 50 \mu \mathrm{m}$ & $0.75 \times 2 \times 2 \mu \mathrm{m}$ & 1 small $+20 \mathrm{big}$ & 40 small & $16 \mathrm{~s}$ & $30 \mathrm{~s}$ & $1.875 \mathrm{X}$ \\
a.2: tower & $39 \times 40 \mu \mathrm{m}$ & $0.75 \times 1 \times 1 \mu \mathrm{m}$ & 5 small $+26 \mathrm{big}$ & 52 small & $17 \mathrm{~s}$ & $33 \mathrm{~s}$ & $1.941 \mathrm{X}$ \\
a.3: staircase & $11.25 \times 40 \mu \mathrm{m}$ & $0.75 \times 5 \times 2 \mu \mathrm{m}$ & 4 small $+7 \mathrm{big}$ & 15 small & $9 \mathrm{~s}$ & $15 \mathrm{~s}$ & $1.666 \mathrm{X}$ \\
\hline
\end{tabular}

The structures were microfabricated multiple times (16 times each structure of type A and 6 times each structure of type B), setting up the 3D printer and executing the GWL scripts, then developed, metalized by gold sputtering, and examined in an electronic microscope.

The fabrication times were measured using the activity log file of the $3 \mathrm{D}$ printer, which had one-second resolution; for each different configuration, all fabrication times were consistent to the second for structures printed in 15 seconds or less, and within \pm 1 second (with very small variability, as almost all prints had the same printing time) for structures that took longer. In all cases, structures of type A were faster to print than ones of type B, as shown in the sixth to eigth columns of Table 1. In the table, there is a row for each one of the three types of structures. For each structure, its overall nominal dimensions are provided in the second column (the structures have identical width and depth, if the overhangs of the staircase are ignored), and the nominal dimensions of its overhanging features in the third column (for the filter, the width and depth are the nominal diameter of the holes). The fourth column shows the numbers of slices in each resolution for configurations of type A, expressed as the sum of numbers of lowresolution (big) and high-resolution (small) slices. The fifth column shows the numbers of slices that are required when printing only at high-res, i.e. type B. The sixth and seventh columns show printing times for configurations of types A and B respectively. Finally, the eighth column shows the effective speed-up for each structure.

As an additional test, the GWL scripts for structures of type A were modified to print big voxels for all toolpaths, even the ones marked by the algorithm as high resolution toolpaths (Figure 3, row d). In this configuration, the geometry of the honeycomb of the filter was significantly modified (the effective diameter of the holes of the printed filter shrunk and their vertical aspect ratio increased). The staircase became uneven, although this is an artifact of the printing configuration: staircase evenness is a $2.5 \mathrm{D}$ feature, and can be perfectly reproduced at low resolutions with adaptive slicing. Also, the overhangs of the staircase became significantly thicker (but this cannot be solved with adaptive slicing). The overhangs of the tower were completely fused together.

\section{Discussion}

In the experimental setup described in the previous sections, the minimal voxel has approximately half the volume and height of the maximal voxel, so consequently the maximal theoretical speed-up is $2 X$. Note, however, that much larger size ranges have been reported, with height ranges from $0.6 \mu \mathrm{m}$ to $15 \mu \mathrm{m}$ (Lim et al., 2006). Using MLM with this size range would allow for a dramatically larger maximal speed-up, up to $25 \mathrm{X}$ (or even larger, under the assumption that the tallest voxels are also several times wider than the shortest ones, and printing speeds were uniform for all voxel sizes). It would also make sense to define more than two voxel sizes, in order to maximize effective speed-ups. The effective speed-up, on the other hand, depends on the geometry of the 
3D structure to be printed: the speed-up will be higher if most of the shape can be printed at low resolutions with relatively few details at higher resolution.

TPP is commonly used to prototype microstructures for academic and industrial research, but currently it is too slow for many potential applications: in some cases, the structures to be fabricated are relatively large but require very high-resolution features; for example microfluidics devices with sub-micrometer features, such as very fine filters (as the one presented in Figure 2, a.1). Because TPP is so slow, most microfluidics devices are manufactured with other technologies (working around their limitations), while metamaterials and surfaces with engineered wettability (which often require manufacturing of very small overhanging features embedded in a lowerresolution bulk, such as the objects presented in Figure 2, a.2 and a.3) are commonly produced with patterning and/or self-assembly processes. Adaptive slicing can be used to accelerate TPP at very high resolutions, but it cannot accurately reproduce structures with very thin overhangs, as MLM does. Usually, research is conducted in a cyclic way, iterating alternatively over hypotheses and experimental designs to test these hypotheses. MLM can accerelate this hypothesis-testing cycle, by reducing the time required to manufacture 3D devices with TPP.

Therefore, MLM can open new research opportunities where it was previously infeasible to manufacture extremely large structures with sub-micrometer resolution, such as in the case of surface wetting: wetting experiments require surface samples of at least around one square centimeter, which may take from days to weeks to manufacture with TPP at the highest resolution. Ultimately, MLM enables the application of TPP to new problems, in ways that were previously impractical.

The experiments described here have been performed with a commercial TPP printer, but the software can be easily adapted to generate toolpaths for any other TPP printer. Additionally, MLM, the underlying algorithm, is generic enough that it can be adapted to work with hybrid AM, were two or more different AM technologies are used together. Much work has been devoted to solve the technical hurdles to combine layered manufacturing technologies at multiple resolution levels, as described in Section 1.3, but little to automatize hybrid layered manufacturing through the use of specialized software. MLM can be easily extended to automatically plan toolpaths for hybrid combinations of additive and subtractive layered manufacturing, overprinting first with a low resolution additive process, then removing the excess material with a high resolution subtractive process, or vice versa.

\section{Conclusion}

Devices and technological processes rely more and more on software to control or operate them. In doing so, software becomes part of the forces pushing the boundaries of what is possible to do in technology and science. However, as this software becomes ever more complex and is applied to similar technologies, it can also become an almost invisible prison that constrains people to think and use technology within a rigid mindset.

This is the case for slicing software: at its core, any non-trivial slicing software implementation is a complex pipeline of computational geometry operations. It is difficult to implement new features on top of it, such as trying to take advantage of the characteristics of a specific manufacturing technology. Most users and researchers just use the slicing software provided by commercial providers of cutting-edge AM machines, which typically cannot be modified to incorporate new ideas or algorithms. Meanwhile, 
new algorithms that improve the effectiveness of a technology can open new applications that were previously unfeasible. For the purposes of this work, only a specific part of Nanoscribe's software pipeline had to be modified, but as the source code is not available, a new software pipeline had to be implemented (mostly from scratch, except for the parts reused from Slic3r), consequently renouncing to optimizations made by Nanoscribe to tune their slicing software to their hardware. However, this also means that the implementation of MLM presented here can be more easily adapted to work in other TPP printers and hybrid manufacturing systems. The implementation is publicly available ${ }^{3}$.

In this work, a new slicing algorithm has been presented, which can be used to leverage the ability of TPP to easily work at several resolution levels, in order to minimize printing times while still being able to print small features at the highest possible resolution, without the need to manually define the toolpaths. It may also be applied to hybrid layered manufacturing technologies.

\section{Acknowledgements}

The first author has been supported by NANO3D, a BEWARE Fellowship from the Walloon Region, Belgium. The research has been partially funded by the Interuniversity Attraction Poles Programme (IAP 7/38 MicroMAST) from the Belgian Science Policy Office, the Walloon Region and the FNRS. We thank the BEAMS department of the University of Brussels, and specifically Pierre Lambert, Youen Vitry and Sam Dehaeck, who let us use their 3D printer and provided invaluable assistance. The first author also thanks his wife Elisa and his children Isabel and Jose, who brought out the best of himself.

\section{References}

Balčiūnas, E., Lukoševičius, L., Mackevičiūtè, D., Rekštytė, S., Rutkūnas, V., Paipulas, D., Stankevičiūtė, K., Baltriukienė, D., Bukelskienè, V., Piskarskas, A. P. et al. (2014), "Combination of thermal extrusion printing and ultrafast laser fabrication for the manufacturing of $3 \mathrm{~d}$ composite scaffolds", in Frontiers in Ultrafast Optics: Biomedical, Scientific, and Industrial Applications, International Society for Optics and Photonics, pp. 89721N-89721N.

Chu, W.-S., Kim, C.-S., Lee, H.-T., Choi, J.-O., Park, J.-I., Song, J.-H., Jang, K.-H. and Ahn, S.-H. (2014), "Hybrid manufacturing in micro/nano scale: a review", Int. J. Precis. Eng. Man. Vol. 1 No. 1, pp. 75-92.

De Coninck, J., Dunlop, F. and T., H. (2015), "Wetting in $1+1$ dimensions with twoscale roughness", Physica A No. 438, pp. 398-415.

Delattre, B., Bai, H., Ritchie, R., De Coninck, J. and Tomsia, A. (2014), “Unidirectional freezing of ceramic suspensions: In situ X-ray investigation of the effects of additives", ACS Appl. Mater. Interfaces No. 6, pp. 159-166.

Eschenbaum, C., Großmann, D., Dopf, K., Kettlitz, S., Bocksrocker, T., Valouch, S. and Lemmer, U. (2013), "Hybrid lithography: Combining uv-exposure and two photon direct laser writing”, Opt. Express Vol. 21 No. 24, pp. 29921-29926.

\footnotetext{
${ }^{3}$ https://github.com/jdfr/MLM
} 
Gittard, S. D., Nguyen, A., Obata, K., Koroleva, A., Narayan, R. J. and Chichkov, B. N. (2011), "Fabrication of microscale medical devices by two-photon polymerization with multiple foci via a spatial light modulator", Biomed. Opt. Express Vol. 2 No. 11, pp. 3167-3178.

Hildebrand, K., Bickel, B. and Alexa, M. (2013), "Orthogonal slicing for additive manufacturing”, Comput. Graph. Vol. 37 No. 6, pp. 669-675.

Huang, S., Liu, P., Mokasdar, A. and Hou, L. (2013), "Additive manufacturing and its societal impact: a literature review”, Int. J. Adv. Manuf. Tech. Vol. 67, pp. 1191-203.

Jonušauskas, L. and Malinauskas, M. (2014), "Mesoscale 3d manufacturing: varying focusing conditions for efficient direct laser writing of polymers", in SPIE Photonics Europe, International Society for Optics and Photonics, pp. 912620-912620.

Li, Y.-C., Cheng, L.-C., Chang, C.-Y., Lien, C.-H., Campagnola, P. J. and Chen, S.-J. (2012), "Fast multiphoton microfabrication of freeform polymer microstructures by spatiotemporal focusing and patterned excitation”, Opt. Express Vol. 20 No. 17, pp. 19030-19038.

Liao, C.-Y., Bouriauand, M., Baldeck, P. L., Léon, J.-C., Masclet, C. and Chung, T.-T. (2007), "Two-dimensional slicing method to speed up the fabrication of micro-objects based on two-photon polymerization", Appl. Phys. Lett. Vol. 91 No. 3, pp. 033108.

Lim, T. W., Park, S. H., Yang, D.-Y., Kong, H. J. and Lee, K.-S. (2006), “Direct singlelayered fabrication of 3d concavo-convex patterns in nano-stereolithography", Appl. Phys. A Vol. 84 No. 4, pp. 379-383.

Lim, T. W., Son, Y., Yang, D.-Y., Kong, H.-J. and Lee, K.-S. (2011), "Selective ablation-assisted two-photon stereolithography for effective nano-and microfabrication", Appl. Phys. A Vol. 103 No. 4, pp. 1111-1116.

Lim, T. W., Son, Y., Yang, D.-Y., Kong, H.-J., Lee, K.-S. and Park, S. H. (2008), "Highly effective three-dimensional large-scale microfabrication using a continuous scanning method", Appl. Phys. A Vol. 92 No. 3, pp. 541-545.

Ma, W. and He, P. (1999), "An adaptive slicing and selective hatching strategy for layered manufacturing”, Journal of Materials Processing Technology J. Mater Process. Tech. Vol. 89, pp. 191-197.

Malinauskas, M., Rekštytė, S., Lukoševičius, L., Butkus, S., Balčiūnas, E., Peciukaityte, M., Baltriukiene, D., Bukelskiene, V., Butkevicius, A., Kucevicius, P., Rutkunas, V. and Juodkazis, S. (2014), "3d microporous scaffolds manufactured via combination of fused filament fabrication and direct laser writing ablation", $\mathrm{Mi}$ cromachines Vol. 5, pp. 839-858.

Matsuo, S., Juodkazis, S. and Misawa, H. (2005), "Femtosecond laser microfabrication of periodic structures using a microlens array", Appl. Phys. A Vol. 80 No. 4, pp. 683685.

Pandey, P. M., Reddy, N. V. and Dhande, S. G. (2003), "Slicing procedures in layered manufacturing: a review", Rapid Prototyping J. Vol. 9 No. 5, pp. 274-288. 
Park, S. H., Lee, S. H., Yang, D.-Y., Kong, H. J. and Lee, K.-S. (2005), "Subregional slicing method to increase three-dimensional nanofabrication efficiency in two-photon polymerization", Appl. Phys. Lett. Vol. 87 No. 15, pp. 154108.

Ranellucci, A. (2017). http://slic3r.org/.

Sabourin, E., Houser, S. A. and Bøhn, J. H. (1996), "Adaptive slicing using stepwise uniform refinement”, Rapid Prototyping Journal Vol. 2, pp. 20-26.

Spangenberg, A., Hobeika, N., Stehlin, F., Malval, J.-P., Wieder, F., Prabhakaran, P., Baldeck, P. and Soppera, O. (2013), Updates in Advanced Lithography, Intech, chapter Recent Advances in Two-Photon Stereolithography, pp. 35-63.

Sun, H.-B. and Kawata, S. (2004), NMR - 3D Analysis · Photopolymerization, Springer, chapter Two-Photon Photopolymerization and 3D Lithographic Microfabrication, pp. 169-273.

Sun, H.-B., Takada, K., Kim, M.-S., Lee, K.-S. and Kawata, S. (2003), "Scaling laws of voxels in two-photon photopolymerization nanofabrication", Applied Physics Letters Vol. 83 No. 6, pp. 1104-1106.

Xiong, W., Zhou, Y. S., He, X. N., Gao, Y., Mahjouri-Samani, M., Jiang, L., Baldacchini, T. and Lu, Y. F. (2012), "Simultaneous additive and subtractive threedimensional nanofabrication using integrated two-photon polymerization and multiphoton ablation", Light: Science \& Applications Vol. 1 No. 4, pp. e6.

Zhu, Z., Dhokia, V., Nassehi, A. and Newman, S. T. (2013), "A review of hybrid manufacturing processes-state of the art and future perspectives", Int. J. Comp Integ. M. Vol. 26 No. 7, pp. 596-615. 